\title{
Questionário de Atividades de Autocuidado com o Diabetes: tradução, adaptação e avaliação das propriedades psicométricas
}

\author{
Questionnaire of Diabetes Self-Care Activities: translation, \\ cross-cultural adaptation and evaluation of psychometric properties
}

Murilo José Michels', Marisa Helena César Coral'2, Thiago Mamôru Sakae', Tanise Balvedi Damas ${ }^{3}$, Letícia Maria Furlanetto ${ }^{1,2}$

${ }^{1}$ Programa de Pós-Graduação em Ciências Médicas, Universidade Federal de Santa Catarina (PPGCM) UFSC), Florianópolis, SC, Brasil 2 Departamento de Clínica Médica, UFSC, Florianópolis, SC, Brasil ${ }^{3}$ Ambulatório de Endocrinologia, Hospital Universitário, UFSC, Florianópolis, SC, Brasil
Correspondência para: Murilo José Michels

Rodovia João Paulo, 614/705 88030-300 - Florianópolis, SC Brasil

murilomichels@yahoo.com.br

Recebido em 2/Fev/2010 Aceito em 31/Ago/2010

\section{RESUMO}

Objetivos: Traduzir para o português, adaptar culturalmente e avaliar as propriedades psicométricas do Summary of Diabetes Self-Care Activities Questionnaire - SDSCA. Materiais e métodos: $O$ processo seguiu as normas internacionais para adaptação e avaliação das propriedades psicométricas. $\mathrm{O}$ alfa de Cronbach $(\alpha)$ foi utilizado para avaliar a consistência interna (correlação interitens) e a confiabilidade (teste-reteste e correlação interavaliador). Resultados: A correlação interitens variou de $\alpha=0,09$ a $\alpha=0,86$. Na avaliação teste-reteste, a menor correlação foi para o item "ingerir doces" $(\alpha=0,15)$ e a maior, para os itens sobre o uso do cigarro $(\alpha=$ $1,00)$. As correlações interavaliador variaram de $\alpha=0,29$ a $\alpha=1,00$. Conclusões: 0 questionário adaptado apresentou propriedades psicométricas semelhantes às do SDSCA. Sua versão para o Brasil fornece um questionário confiável e válido para avaliar a aderência ao autocuidado nos diabéticos em nosso meio. Arq Bras Endocrinol Metab. 2010;54(7):644-51

\section{Descritores}

Diabetes melito; autocuidado; estudos de validação; tradução (processo)

\begin{abstract}
Objectives: To translate into Portuguese, perform cross-cultural adaptation and to evaluate the psychometric properties of the Summary of Diabetes Self-Care Activities Questionnaire - SDSCA. Materials and methods: The process followed the international guidelines for the adaptation and evaluation of psychometric properties. The Cronbach's alpha $(\alpha)$ was determined to evaluate the internal consistency (inter-itens correlation) and the reliability (test-retest and interevaluator correlation). Results: The inter-itens correlation showed values of $\alpha=0.09$ to $\alpha=0.86$. In the test-retest evaluation, the lowest correlation was obtained for the item "eat sweets" $(\alpha=$ $0.15)$ and the highest correlation was obtained for the items concerning smoking $(\alpha=1.00)$. The inter-evaluator correlations varied from $\alpha=0.29$ to $\alpha=1.00$. Conclusions: The adapted questionnaire showed psychometric properties similar to those of the SDSCA. Its Brazilian version provides a reliable and valid questionnaire to evaluate diabetic patient adherence to self-care in our community. Arq Bras Endocrinol Metab. 2010;54(7):644-51
\end{abstract}

Keywords

Diabetes mellitus; self care; validation studies; translating (process)

\section{INTRODUÇ̃̃O}

A aderência ao tratamento no diabetes melito (DM) é um fator essencial para controle da glicemia e redução da incidência das complicações (1-3). Para o trata- mento do DM, além do uso da medicação, são necessárias diversas atividades de autocuidado como o seguimento de um plano alimentar, a monitorização da glicemia e a realização de atividades físicas. Essas atividades são vis- 
tas atualmente como o ponto central do tratamento dos diabéticos $(1,4)$. A avaliação da aderência ao tratamento dos diabéticos é normalmente realizada, nas consultas, pelas avaliações clínica e laboratorial desses pacientes (1). Ter instrumentos que permitam avaliar e medir essa aderência é importante para aplicação em pesquisas e também para guiar os clínicos em sua avaliação.

Medir a aderência no DM é difícil devido à complexidade do regime terapêutico, que envolve as diferentes atividades de autocuidado (4-6). Além disso, para avaliar a aderência ao tratamento em pesquisas, é necessário ter instrumentos confiáveis e válidos $(4,7)$. Diversas medidas têm sido utilizadas para essa avaliação, como a hemoglobina glicada ( $\mathrm{HbAlc})$, a contagem da medicação e o relato dos pacientes $(1,6)$. $\mathrm{O}$ autorrelato a partir de perguntas específicas em entrevistas ou questionários tem se mostrado uma das abordagens mais práticas e efetivas para avaliar a aderência aos cuidados com o diabetes $(4,6)$.

Dos questionários utilizados para avaliar a aderência ao autocuidado nos diabéticos, o Summary of Diabetes Self-Care Activities Questionnaire (SDSCA) $(4,6)$ tem sido um dos instrumentos mais usados em pesquisa (8-10). Foi desenvolvido para avaliar, de maneira sistematizada, a aderência às atividades de autocuidado no paciente diabético $(4,6)$. Pode também ser útil para guiar os clínicos na avaliação e no seguimento dos pacientes. O SDSCA questiona a realização de atividades pelos pacientes e sua concordância com a prescrição médica ou de outro profissional de saúde $(4,6)$. Sua validade e confiabilidade já foram avaliadas e estabelecidas em populações norte-americanas de língua inglesa $(4,6)$ e espanhola $(7)$ e também em portugueses $(5)$.

O SDSCA avalia cinco aspectos do regime de tratamento do diabetes, agrupados em seis dimensões do autocuidado: alimentação (geral e específica), atividade física, uso da medicação, monitorização da glicemia e o cuidado com os pés, avaliando também o tabagismo (6). As dimensões representam atividades distintas do tratamento do diabetes, realizadas de maneira independente pelos pacientes $(4,6)$. O questionário original $(6)$ foi revisado e modificado a partir da avaliação de sete estudos que o utilizaram na avaliação dos pacientes (4). O SDSCA revisado possui 12 itens para medir os componentes do autocuidado no DM, apresentando, também, outros 14 itens adicionais que podem ser utilizados para investigação mais detalhada de alguns desses cuidados $(4,7)$.

Quando escalas ou questionários são utilizados em países diferentes daqueles de seu desenvolvimento, é necessária mais do que a simples tradução literal, em razão das diferenças culturais, semânticas e idiomáticas que precisam ser revistas (11-13). Assim, além da tradução, deve-se realizar uma adaptação transcultural e também a avaliação das propriedades psicométricas do novo questionário $(7,11,13)$. Nesse sentido, o objetivo deste estudo é traduzir para o português, adaptar culturalmente e avaliar as propriedades psicométricas do SDSCA.

\section{SUJEITOS E MÉTODOS}

\section{Participantes}

Ao todo foram selecionados 105 pacientes com DM tipo 2 (DM2) com idade igual ou superior a 30 anos, consecutivamente atendidos no Ambulatório de Endocrinologia do Hospital Universitário da Universidade Federal de Santa Catarina (HU-UFSC). Destes, sete foram excluídos por impossibilidade física ou recusa, contendo a amostra 98 pacientes. Os dados foram coletados de março de 2008 a maio de 2009.

Os pacientes foram caracterizados de acordo com o gênero, a idade, a escolaridade, o estado civil, a renda familiar e o tempo de diagnóstico do diabetes. Foram também avaliados os últimos exames de $\mathrm{HbAlc} \mathrm{realiza-}$ dos até três meses anteriores à consulta.

Este estudo foi aprovado pelo Comitê de Ética em Pesquisa em Seres Humanos da UFSC sob o $\mathrm{n}^{\circ}$ 008/2008. Todos os pacientes assinaram o consentimento livre e esclarecido.

\section{Tradução e adaptação transcultural}

O processo de tradução e adaptação do questionário seguiu os procedimentos recomendados internacionalmente para a tradução e adaptação de instrumentos de pesquisa (11-14). A tradução e a adaptação foram autorizadas pela autora principal do SDSCA (Deborah E. Toobert ).

Inicialmente foi avaliada a validade de face (11-14) do SDSCA por um endocrinologista (M.H.C.C.) e dois psiquiatras (L.M.F. e M.J.M.), para verificar se os itens, em seu aspecto geral, avaliavam os conceitos desejados. Em seguida, foi realizada a tradução do questionário por um professor de inglês. Optou-se por utilizar, além do SDSCA revisado (Revised SDSCA Scale) (4), alguns itens da versão expandida (Expanded Version of the SDS$C A$ ) (4) como um item do cuidado com os pés e os itens sobre o uso da medicação e também o tabagismo, considerados importantes para avaliar o autocuidado dos pacientes em nosso meio. $\mathrm{O}$ item sobre o consumo 
de carboidratos do SDSCA original (6) foi mantido, em vez do item sobre espaçamento de carboidratos do SDSCA revisado (4), por estar mais em acordo com as recomendações dadas aos pacientes diabéticos pelos endocrinologistas na nossa realidade.

Foram realizadas as equivalências semântica e conceitual (11-14). Para efetuar as equivalências, foram avaliados os significados das palavras de cada item e se as sentenças representavam os mesmos conceitos nas diferentes culturas em questão (11-14). A seguir, no desdobramento cognitivo, questionou-se a dez pacientes com DM2, atendidos no Ambulatório de Endocrinologia do HU-UFSC, sobre seu entendimento de cada item do questionário, com o objetivo de avaliar a clareza e a compreensão da terminologia. O desdobramento cognitivo incluiu: "Você tem dificuldade para responder esta pergunta?", "Você achou alguma palavra difícil de entender?", "Você faria esta pergunta de outra maneira?", "Você acha que está faltando alguma coisa na pergunta?". E sobre a impressão geral do questionário: "É claro, fácil de entender e fácil de responder?" (Se não, por quê?). "É muito grande?", "Você deseja fazer algum comentário sobre o questionário?". As respostas dos pacientes foram analisadas pelos avaliadores do questionário, sendo realizadas readaptações em alguns itens. O questionário adaptado foi aplicado em 20 pacientes do mesmo ambulatório, em um préteste, para avaliar aceitabilidade, compreensão e impressões. Os itens do SDSCA e sua tradução e adaptação são mostrados no Anexo 1 .

Seguiu-se a retrotradução do questionário para o inglês por um americano fluente em inglês e português, sendo este depois enviado para a autora principal. A autora ratificou o questionário retrotraduzido referindo que este mantinha o sentido e os conceitos da avaliação do questionário original.

O questionário traduzido e adaptado para o Brasil foi denominado "Questionário de Atividades de $\mathrm{Au}$ tocuidado com o Diabetes" (QAD). O QAD possui seis dimensões e 15 itens de avaliação do autocuidado com o diabetes: "alimentação geral" (com dois itens), "alimentação específica" (três itens), "atividade física" (dois itens), "monitorização da glicemia" (dois itens), "cuidado com os pés" (três itens) e "uso da medicação" (três itens, utilizados de acordo com o esquema medicamentoso). Além disso, possui outros três itens para a avaliação do tabagismo (Anexo 2). Quando avaliados com o questionário, os pacientes relatam com que frequência eles realizaram as atividades ou os comportamentos nos sete dias anteriores. As respostas variam de
0 a 7, com os escores indicando as performances das atividades de autocuidado $(4,6)$.

\section{Aplicação do Questionário de Atividades de Autocuidado com 0 Diabetes (QAD)}

Após as etapas anteriores, o questionário foi aplicado em 98 pacientes com DM2, atendidos no mesmo ambulatório, para avaliar as propriedades psicométricas. Cada item do questionário foi lido pelo pesquisador, que anotou a resposta do paciente para cada um deles. A aplicação do questionário levou entre sete e 15 minutos por paciente. Dez pacientes foram avaliados por dois pesquisadores independentes (M.J.M. e T.B.D.) para realizar a correlação interavaliador. Em 15 pacientes que aceitaram retornar foi repetida a aplicação do questionário após uma semana para avaliar a validade teste-reteste.

\section{Análise estatística}

O questionário traduzido e adaptado foi aplicado nos pacientes para a avaliação de seu comportamento na amostra. Para a análise da aderência aos itens do questionário, estes foram parametrizados em dias por semana, de 0 a 7 , sendo zero a situação menos desejável e sete a mais favorável. Nos itens da dimensão alimentação específica que questionam sobre o consumo de alimentos ricos em gordura e doces, os valores foram invertidos (se $7=0,6=1,5=2,4=3,3=4,2=5$, $\mathrm{l}=6,0=7$ e vice-versa), como sugerido no SDSCA revisado (4). A avaliação do tabagismo foi codificada considerando-se a proporção de fumantes, a média de cigarros consumidos e a última vez em que fumou.

Como a aderência ao diabetes é multidimensional, a consistência interna foi avaliada para cada dimensão do autocuidado. $\mathrm{O}$ alfa de Cronbach $(\alpha)$ foi utilizado para avaliar a consistência interna (correlação interitens nas dimensões do autocuidado) e a confiabilidade (testereteste e correlação interavaliador). Para a análise da correlação interitens, considerou-se como fraca uma correlação correspondente a um coeficiente $\alpha$ inferior a 0,30 , moderada entre 0,30 e 0,70 e forte se superior a $0,70(4,5)$. Foram considerados estatisticamente significativos os valores de $\mathrm{p} \leq 0,05$. Foram descritas as características sociodemográficas e clínicas da amostra. As variáveis contínuas foram descritas como médias e desvios-padrão (DP) e as variáveis categóricas, como frequências e porcentagens. Para a análise estatística, foi utilizado o software SPSS for windows, versão 10 (SPSS Inc., Chicago, IL, Estados Unidos) (15). 
Anexo 1

\begin{tabular}{l}
\hline Itens do SDSCA \\
\hline Diet (general) \\
\hline How many of the last SEVEN DAYS have you followed a healthful eating plan? \\
On average, over the past month, how many DAYS PER WEEK have you followed \\
your eating plan?
\end{tabular}

Tradução e adaptação

Alimentação Geral

1.1 Em quantos dos últimos SETE DIAS seguiu uma dieta saudável?

1.2 Durante o último mês, QUANTOS DIAS POR SEMANA, em média, seguiu a orientação alimentar, dada por um profissional de saúde (médico, enfermeiro, nutricionista)?

\section{Diet (specific)}

\section{Alimentação especifica}

On how many of the last SEVEN DAYS did you eat five or more servings of fruits and/or vegetables?

2.1 Em quantos dos últimos SETE DIAS comeu cinco ou mais porções de frutas e/ ou vegetais?

On how many of the last SEVEN DAYS did you eat high fat foods such as red meat or full-fat dairy products?

2.2 Em quantos dos últimos SETE DIAS comeu alimentos ricos em gordura como carnes vermelhas ou derivados de leite integral?

On how many of the last 7 days did your meals include sweets?

\section{Exercise}

2.3 Em quantos dos últimos SETE DIAS comeu doces?

On how many of the last SEVEN DAYS did you participate in at least 30 minutes of physical activity? (Total minutes of continuous activity, including walking).

\section{Atividade física}

3.1 Em quantos dos últimos SETE DIAS realizou atividade física durante pelo menos 30 minutos? (minutos totais de atividade contínua, inclusive andar).

On how many of the last SEVEN DAYS did you participate in a specific exercise session (such as swimming, walking, biking) other than what you do around the house or as part of your work?

\section{Blood sugar testing}

On how many of the last SEVEN DAYS did you test your blood sugar?

3.2 Em quantos dos últimos SETE DIAS praticou algum tipo de exercício físico específico (nadar, caminhar, andar de bicicleta), sem incluir suas atividades em casa ou em seu trabalho?

\section{Monitorização da glicemia}

4.1 Em quantos dos últimos SETE DIAS avaliou o açúcar no sangue?

On how many of the last SEVEN DAYS did you test your blood sugar the number of times recommended by your health care provider?

Foot care
On how many of the last SEVEN DAYS did you check your feet?
On how many of the last SEVEN DAYS did you inspect the inside of your shoes?

On how many of the last SEVEN DAYS did you dry between your toes after washing?

Medications
On how many of the last SEVEN DAYS, did you take your recommendeddiabetes
medication? OR
On how many of the last SEVEN DAYS did you take your recommended insulin
injections?
On how many of the last SEVEN DAYS did you take your recommended number of
diabetes pills?

\section{Smoking}

Have you smoked a cigarette - even one puff - during the past SEVEN DAYS?

0. No 1. Yes.

If yes, how many cigarettes did you smoke on an average day?

Number of cigarettes:

When did you last smoke a cigarette?

- More than two years ago, or never smoked

- One to two years ago

- Four to twelve months ago

- One to three months ago

- Within the last month

- Today
4.2 Em quantos dos últimos SETE DIAS avaliou o açúcar no sangue a quantidade de vezes recomendada pelo médico ou enfermeiro?

\section{Cuidados com os pés}

5.1 Em quantos dos últimos SETE DIAS examinou os seus pés?

5.2 Em quantos dos últimos SETE DIAS examinou dentro dos sapatos, antes de calçá-los?

5.3 Em quantos dos últimos SETE DIAS secou os espaços entre os dedos dos pés depois de lavá-los?

\section{Medicação}

6.1 Em quantos dos últimos SETE DIAS tomou seus medicamentos do diabetes, conforme recomendado? OU (se insulina e comprimidos):

6.2 Em quantos dos últimos SETE DIAS, tomou suas injeções de insulina,

6.3 Em quantos dos últimos SETE DIAS tomou o número indicado de comprimidos do diabetes?

\section{Tabagismo}

7.1 Você fumou um cigarro - ainda que só uma tragada - durante os últimos sete dias? $\square$ Não $\square \operatorname{Sim}$

7.2 Se sim, quantos cigarros fuma, habitualmente, num dia? Número de cigarros:

7.3 Quando fumou o seu último cigarro?

$\square$ Nunca fumou

$\square$ Há mais de dois anos atrás

$\square$ Um a dois anos atrás

$\square$ Quatro a doze meses atrás

$\square$ Um a três meses atrás

$\square$ No último mês

$\square$ Hoje conforme recomendado? 
Anexo 2. Questionário de atividades de autocuidado com o diabetes - QAD

(As perguntas que se seguem questionam-no sobre seus cuidados com o diabetes durante os últimos sete dias. Se você esteve doente durante os últimos sete dias, por favor lembre-se dos últimos sete dias em que não estava doente)

\begin{tabular}{|c|c|c|c|c|c|c|c|c|}
\hline 1. ALIMENTAÇÃO GERAL & & & & & & & & \\
\hline 1.1 Em quantos dos últimos SETE DIAS seguiu uma dieta saudável? & 0 & 1 & 2 & 3 & 4 & 5 & 6 & 7 \\
\hline $\begin{array}{l}\text { 1.2 Durante o último mês, QUANTOS DIAS POR SEMANA, em média, seguiu a orientação alimentar, } \\
\text { dada por um profissional de saúde (médico, enfermeiro, nutricionista)? }\end{array}$ & 0 & 1 & 2 & 3 & 4 & 5 & 6 & 7 \\
\hline 2. ALIMENTAÇÃO ESPECÍFICA & & & & & & & & \\
\hline 2.1 Em quantos dos últimos SETE DIAS comeu cinco ou mais porções de frutas e/ou vegetais? & 0 & 1 & 2 & 3 & 4 & 5 & 6 & 7 \\
\hline 2.2 Em quantos dos últimos SETE DIAS comeu alimentos ricos em gordura, como carnes vermelhas & & & & & & & & \\
\hline ou alimentos com leite integral ou derivados? & 0 & 1 & 2 & 3 & 4 & 5 & 6 & 7 \\
\hline 2.3 Em quantos dos últimos sete dias comeu doces? & 0 & 1 & 2 & 3 & 4 & 5 & 6 & 7 \\
\hline 3. ATIVIDADE FÍSICA & & & & & & & & \\
\hline 3.1 Em quantos dos últimos SETE DIAS realizou atividade física durante pelo menos 30 minutos & & & & & & & & \\
\hline (minutos totais de atividade contínua, inclusive andar)? & 0 & 1 & 2 & 3 & 4 & 5 & 6 & 7 \\
\hline 3.2 Em quantos dos últimos SETE DIAS praticou algum tipo de exercício físico específico & & & & & & & & \\
\hline (nadar, caminhar, andar de bicicleta), sem incluir suas atividades em casa ou em seu trabalho? & 0 & 1 & 2 & 3 & 4 & 5 & 6 & 7 \\
\hline 4. MONITORIZAÇÃO DA GLICEMIA & & & & & & & & \\
\hline 4.1 Em quantos dos últimos SETE DIAS avaliou o açúcar no sangue? & 0 & 1 & 2 & 3 & 4 & 5 & 6 & 7 \\
\hline 4.2 Em quantos dos últimos SETE DIAS avaliou o açúcar no sangue o número de vezes recomendado & & & & & & & & \\
\hline pelo médico ou enfermeiro? & 0 & 1 & 2 & 3 & 4 & 5 & 6 & 7 \\
\hline 5. CUIDADOS COM OS PÉS & & & & & & & & \\
\hline 5.1 Em quantos dos últimos SETE DIAS examinou os seus pés? & 0 & 1 & 2 & 3 & 4 & 5 & 6 & 7 \\
\hline 5.2 Em quantos dos últimos SETE DIAS examinou dentro dos sapatos antes de calçá-Ios? & 0 & 1 & 2 & 3 & 4 & 5 & 6 & 7 \\
\hline 5.3 Em quantos dos últimos SETE DIAS secou os espaços entre os dedos dos pés depois de lavá-los? & 0 & 1 & 2 & 3 & 4 & 5 & 6 & 7 \\
\hline 6. MEDICAÇÃO & & & & & & & & \\
\hline 6.1 Em quantos dos últimos SETE DIAS tomou seus medicamentos do diabetes, conforme foi recomend & & & & & & & & \\
\hline OU (se insulina e comprimidos): & 0 & 1 & 2 & 3 & 4 & 5 & 6 & 7 \\
\hline 6.2 Em quantos dos últimos SETE DIAS tomou suas injeções de insulina, conforme foi recomendado? & 0 & 1 & 2 & 3 & 4 & 5 & 6 & 7 \\
\hline 6.3 Em quantos dos últimos SETE DIAS tomou o número indicado de comprimidos do diabetes? & 0 & 1 & 2 & 3 & 4 & 5 & 6 & 7 \\
\hline 7. TABAGISMO & & & & & & & & \\
\hline 7.1 Você fumou um cigarro - ainda que só uma tragada - durante os últimos sete dias? $\square$ Não $\square$ Sim & & & & & & & & \\
\hline 7.2 Se sim, quantos cigarros fuma, habitualmente, num dia? Número de cigarros: & & & & & & & & \\
\hline 7.3 Quando fumou o seu último cigarro? & & & & & & & & \\
\hline$\square$ Nunca fumou & & & & & & & & \\
\hline$\square$ Há mais de dois anos atrás & & & & & & & & \\
\hline$\square$ Um a dois anos atrás & & & & & & & & \\
\hline$\square$ Quatro a doze meses atrás & & & & & & & & \\
\hline$\square$ Um a três meses atrás & & & & & & & & \\
\hline$\square$ No último mês & & & & & & & & \\
\hline$\square$ Hoje & & & & & & & & \\
\hline
\end{tabular}

\section{RESULTADOS}

Dos 98 pacientes avaliados, $69,4 \%$ eram do sexo feminino, tinham escolaridade média de $4,9( \pm 3,3)$ anos e apenas seis pacientes referiram morar sozinhos (Tabela 1 ).

No desdobramento cognitivo e avaliação pré-teste, os pacientes relataram suas impressões sobre o questionário. Os relatos incluíram que este era breve e fácil de responder e que os levava a recordar os cuidados necessários com o diabetes. Alguns pacientes mencio- naram ainda que tinham aprendido novos cuidados. Os pacientes referiram que, após serem avaliados pelo questionário, poderiam se cuidar melhor, ficando atentos para atitudes que levariam a uma melhor evolução de sua doença.

A correlação interitens de cada dimensão foi variável com valores de $\alpha=0,09$ a $\alpha=0,86$. A maior correlação foi encontrada para a dimensão "monitorização da glicemia" e a mais baixa, para os itens sobre o uso da medicação (Tabela 2). 
Tabela 1. Dados sociodemográficos e clínicos dos pacientes diabéticos tipo 2

\begin{tabular}{lc}
\hline Características & Pacientes estudados* \\
\hline Sexo feminino, $n$ (\%) & $68(69,4)$ \\
Casados/amasiados, $n(\%)$ & $76(78,4)$ \\
Idade, anos & $59,7 \pm 8,9$ \\
Escolaridade, anos & $4,9 \pm 3,3$ \\
Renda familiar, salários-mínimos & $3,1 \pm 2,7$ \\
Tempo de diagnóstico do diabetes, anos & $11,8 \pm 9,2$ \\
HbA1c & $8,49 \pm 2,25$ \\
Uso de medicações, $n$ (\%) & \\
Sem medicações & $3(3,1)$ \\
Hipoglicemiante oral & $52(53,1)$ \\
Insulina & $7(17,1)$ \\
Hipoglicemiante oral e insulina & $36(36,7)$ \\
\hline * Os valores são descritos em média \pm desvio-padrão, exceto se especificados de outra forma. \\
HbA1c: hemoglobina glicada. \\
Tabela 2. Avaliação da consistência interna das dimensões do QAD (N = 98) \\
\hline Dimensões do autocuidado & Correlação interitem* \\
\hline Alimentação geral & 0,61 \\
Alimentação específica & 0,23 \\
Atividade física & 0,69 \\
Monitorização da glicemia & 0,86 \\
Cuidado com os pés & 0,28 \\
Medicação & 0,09 \\
\hline
\end{tabular}

$* \alpha$ de Cronbach.

QAD: Questionário de Atividades de Autocuidado com o Diabetes.

Os itens relativos à avaliação do tabagismo não tiveram sua consistência interna avaliada por não se referirem à aderência e serem codificados de modo a avaliar a quantidade e a frequência do uso de cigarros.

A correlação teste-reteste de cada item variou de $\alpha=$ 0,15 a $\alpha=1,0$. A menor correlação foi encontrada para o item "ingerir doces" e a maior para os itens "fumar" e "quantidade de cigarros por dia" que apresentaram o valor de $\alpha=1,0$, ou seja, não houve diferença entre as avaliações dos pacientes no intervalo de uma semana (Tabela 3 ). $\mathrm{Na}$ correlação interavaliador, o menor valor foi encontrado para o item "seguir a orientação alimentar" e os maiores para "fumar" e "quantidade de cigarros por dia", que não apresentaram variação entre as entrevistas (Tabela 3).

A avaliação da aderência aos itens do QAD mostra os níveis de aderência às atividades de autocuidado da população avaliada neste estudo e permite a comparação entre as diferentes atividades de autocuidado nos pacientes diabéticos. Nos pacientes avaliados, o menor valor de aderência foi encontrado para o item "realizar atividades físicas específicas (caminhar, nadar, etc.)" (1,24 $\pm 2,78$ dias por semana) e o maior, para o item "tomar injeções de insulina conforme recomendado" ( $6,70 \pm 1,15$ dias por semana) (Tabela 4$)$.
Tabela 3. Avaliação da confiabilidade do QAD

\begin{tabular}{|c|c|c|}
\hline \multirow[b]{2}{*}{ Itens do QAD } & \multicolumn{2}{|c|}{ Correlação } \\
\hline & $\begin{array}{l}\text { Teste-reteste } \\
(n=15)\end{array}$ & $\begin{array}{l}\text { Interavaliador* } \\
\quad(n=10)\end{array}$ \\
\hline 1. Seguir uma dieta saudável & 0,91 & 0,91 \\
\hline 2. Seguir a orientação alimentar & 0,82 & 0,29 \\
\hline $\begin{array}{l}\text { 3. Ingerir cinco ou mais porções de } \\
\text { frutas e/ou vegetais }\end{array}$ & 0,69 & 0,76 \\
\hline $\begin{array}{l}\text { 4. Ingerir carne vermelha e/ou derivados } \\
\text { de leite integral }\end{array}$ & 0,64 & 0,92 \\
\hline 5. Ingerir doces & 0,15 & 0,59 \\
\hline $\begin{array}{l}\text { 6. Realizar atividades físicas por pelo } \\
\text { menos } 30 \text { minutos }\end{array}$ & 0,81 & 0,94 \\
\hline $\begin{array}{l}\text { 7. Realizar atividades físicas específicas } \\
\text { (caminhar, nadar etc.) }\end{array}$ & 0,70 & 0,98 \\
\hline 8. Avaliar o açúcar no sangue & 0,89 & 0,88 \\
\hline $\begin{array}{l}\text { 9. Avaliar } 0 \text { açúcar no sangue o número } \\
\text { de vezes recomendado }\end{array}$ & 0,93 & 0,83 \\
\hline 10. Examinar os seus pés & 0,89 & 0,86 \\
\hline $\begin{array}{l}\text { 11. Examinar dentro dos sapatos antes } \\
\text { de calçá-los }\end{array}$ & 0,77 & 0,86 \\
\hline $\begin{array}{l}\text { 12. Secar os espaços entre os dedos dos } \\
\text { pés depois de lavá-los }\end{array}$ & 0,97 & 0,88 \\
\hline $\begin{array}{l}\text { 13. Tomar injeções de insulina conforme } \\
\text { recomendado }\end{array}$ & 0,81 & 0,52 \\
\hline $\begin{array}{l}\text { 14. Tomar o número indicado de } \\
\text { comprimidos do diabetes }\end{array}$ & 0,94 & 0,78 \\
\hline 15. Fumar & 1,0 & 1,0 \\
\hline 16. Quantidade de cigarros por dia & 1,0 & 1,0 \\
\hline 17. Quando fumou o seu último cigarro & 0,98 & 0,99 \\
\hline
\end{tabular}

$* \alpha$ de Cronbach.

QAD: Questionário de Atividades de Autocuidado com o Diabetes.

Tabela 4. Aderência aos itens do Questionário de Atividades de Autocuidado com 0 Diabetes

\begin{tabular}{ll}
\hline Itens do QAD & Aderência* $^{*}$ \\
\hline 1. Seguir uma dieta saudável & $5,45( \pm 2,33)$ \\
2. Seguir a orientação alimentar & $3,40( \pm 2,89)$ \\
3. Ingerir cinco ou mais porções de frutas e/ou vegetais & $4,92( \pm 2,72)$ \\
4. Ingerir carne vermelha e/ou derivados de leite integral & $3,75( \pm 2,18)$ \\
5. Ingerir doces & $1,53( \pm 2,00)$ \\
6. Realizar atividades físicas por pelo menos 30 minutos & $2,70( \pm 2,76)$ \\
7. Realizar atividades físicas específicas (caminhar, nadar etc.) & $1,24( \pm 2,78)$ \\
8. Avaliar o açúcar no sangue & $1,76( \pm 2,59)$ \\
9. Avaliar o açúcar no sangue o número de vezes recomendado & $1,37( \pm 2,37)$ \\
10. Examinar os seus pés & $3,55( \pm 3,32)$ \\
11. Examinar dentro dos sapatos antes de calçá-los & $4,55( \pm 3,23)$ \\
12. Secar os espaços entre os dedos dos pés depois de lavá-los & $5,50( \pm 2,78)$ \\
13. Tomar injeções de insulina conforme recomendado & $6,70( \pm 1,15)$ \\
14. Tomar o número indicado de comprimidos do diabetes & $6,23( \pm 1,99)$
\end{tabular}

* Média da aderência em dias por semana ( \pm desvio-padrão) para as atividades de autocuidado nos sete dias anteriores.

QAD: Questionário de Atividades de Autocuidado com o Diabetes. 


\section{DISCUSSÃO}

O questionário traduzido e adaptado manteve os conceitos e a avaliação das dimensões que o SDSCA se propõe a avaliar, sendo ratificado pela autora principal (Deborah E. Toobert). Após sua adaptação e avaliação das propriedades psicométricas, mostrou ser um instrumento válido e confiável.

As correlações interitens das dimensões do QAD foram variáveis, dependendo do autocuidado avaliado. As dimensões "alimentação geral" e "atividade física" tiveram correlações interitens consideradas moderadas e a dimensão "monitorização da glicemia" apresentou correlação forte. Por outro lado, as dimensões "alimentação específica", "cuidado com os pés" e "medicação" tiveram correlações interitens fracas.

Nossos resultados foram concordantes com os estudos de validação do questionário original (6) e sua revisão (4) e também suas adaptações $(5,7)$. Assim, tanto no SDSCA original (6) e revisado (4) quanto nos estudos de adaptação do SDSCA para os portugueses (5) e para a língua espanhola (7) as correlações interitens foram moderadas a fortes nas dimensões "alimentação geral", "atividade física" e "monitorização da glicemia" e fraca para a dimensão "alimentação específica". As dimensões "cuidado com os pés" e "medicação" não tiveram suas propriedades avaliadas no SDSCA original, mas, em acordo com o QAD, apresentaram correlações interitens fracas no SDSCA revisado (4) e na adaptação para a língua espanhola (7). Os resultados foram discordantes apenas para a dimensão "cuidado com os pés", que teve correlação interitens moderada na adaptação portuguesa (5), enquanto no QAD a correlação foi baixa.

As correlações teste-reteste do $\mathrm{QAD}$ tiveram valores altos, indicando estabilidade nas avaliações através do tempo. A única exceção foi para o item sobre o consumo de doces, que apresentou bastante variabilidade nas duas avaliações, realizadas com uma semana de intervalo. Os valores de correlação encontrados em nosso estudo foram maiores que os do SDSCA original $(6,9)$, no qual, porém, os pacientes foram reavaliados após seis meses, em estações opostas do ano e após intervenções de tratamento (4). As variações encontradas em nossa pesquisa sugerem que o consumo de alguns grupos de alimentos é bastante variável ao longo do mês.

As correlações interavaliador do QAD mostraram resultados semelhantes nas duas avaliações, com exceção do item "seguir a orientação alimentar", que apresentou uma baixa correlação. Os resultados discrepantes nas avaliações dos dois pesquisadores podem indicar que alguns itens têm compreensão mais difícil pelos entrevistados, ou que esses itens questionam os cuidados de forma mais subjetiva. Podem revelar ainda itens que seriam mais influenciados pela interação entre entrevistado e o pesquisador. Em nosso estudo, os questionários foram aplicados por uma residente de endocrinologia e por um psiquiatra e as respostas dos pacientes a alguns itens tiveram respostas diferentes na avaliação dos entrevistadores. As respostas obtidas sugerem que os pacientes poderiam estar mais preocupados em mostrar melhor aderência ao médico que é responsável pela avaliação do DM do que ao psiquiatra que estava investigando questões relativas às suas dificuldades ou aos "problemas de nervoso". Essas correlações não foram avaliadas nos estudos de validação e adaptação do SDSCA (4-7).

Algumas dificuldades ou limitações devem ser apontadas no QAD. Alguns itens podem ser difíceis de avaliar e/ou interpretar, por representarem conceitos amplos ou não avaliarem a aderência de maneira objetiva, como exemplo pode-se citar o item "seguir uma dieta saudável". Diferenças culturais dentro de um mesmo país, como no Brasil, onde, por exemplo, os hábitos alimentares variam de uma região para a outra, também podem levar a dificuldades de avaliação. É necessário também mencionar que o questionário foi avaliado em pacientes diabéticos tipo 2 do ambulatório de um hospital terciário, assim, em pacientes com outros tipos de diabetes e naqueles atendidos em locais com outras características, como a atenção primária, seu comportamento pode ser diferente e serem necessárias novas avaliações e adaptações. Além das dificuldades citadas, deve-se ainda ressaltar que o $\mathrm{QAD}$ não pode fornecer um escore geral da aderência ao tratamento, o que também ocorre no SDSCA $(4,6)$ e suas adaptações $(5,7)$. Isso porque os questionários avaliam aspectos diversos do autocuidado, que apresentam pouca correlação entre si. Mesmo quando os cuidados semelhantes são agrupados em dimensões, os itens que as compõem podem possuir correlações fracas e, assim, nem todas as dimensões podem ter escores de aderência.

Para a utilização do QAD na avaliação da aderência ao autocuidado cada item é questionado ao paciente, que responde em quantos dos últimos sete dias realizou determinada atividade ou comportamento. Avalia-se, então, a realização da atividade de autocuidado descrita em cada item, ou, em algumas dimensões, pode-se obter um valor médio de aderência dos itens e, assim, um escore dessas dimensões. As seguintes dimensões, que 
mostraram ter boas correlações entre os itens, podem ter um escore de aderência: "alimentação geral" (média do número de dias dos itens 1.1 e 1.2 ), "atividade física" (média do número de dias dos itens 3.1 e 3.2 ), e "monitorização da glicemia" (média do número de dias dos itens 4.1 e 4.2). As demais dimensões do QAD - "alimentação específica", "cuidado com os pés" e "medicação" - devem ter cada item avaliado sempre em separado devido à fraca correlação entre eles. $\mathrm{Na}$ avaliação por itens, obtém-se a aderência a determinado cuidado em dias por semana.

Como resultado da avaliação dos pacientes com o QAD, mostramos, na tabela 4 , a aderência a cada item do autocuidado em dias por semana e apresentamos uma das formas de se avaliar os pacientes com o questionário. Em linhas gerais, os maiores valores de aderência foram encontrados para o uso das medicações e o menor, para a realização de atividades físicas, resultados que foram concordantes com outros estudos como mostrado por Bastos e cols. (5). Discussões mais detalhadas acerca dos níveis de aderência não serão realizadas aqui, por não se tratarem de objetivo deste estudo, mas devem ser abordadas em pesquisas futuras.

Além de seu uso em pesquisas, o QAD pode ter utilidade prática guiando os profissionais de saúde na avaliação da aderência ao autocuidado e sua evolução no tratamento dos diabéticos. A aplicação do questionário é fácil, prática e rápida, e este se encontra disponível para uso público no Anexo 2. É possível, ainda, que sua aplicação ajude a melhorar a aderência, pois mostrou recordar os pacientes sobre os cuidados essenciais em seu tratamento. Após responder ao $\mathrm{QAD}$, os pacientes referiram que tinham se lembrado de cuidados que já haviam aprendido em suas consultas e também acreditavam que haviam aprendido novos cuidados. Questionar aos pacientes sobre a realização dos cuidados com o QAD parece tê-los feito refletir sobre como realizavam os cuidados, e, talvez, a linguagem utilizada no questionário pode ter sido mais acessível em relação à utilizada nas consultas. Sabe-se que a própria pesquisa ou o questionamento podem reforçar os cuidados, o que foi mostrado em estudos recentes, que relataram uma melhora na aderência após a realização das pesquisas que questionavam os pacientes sobre o autocuidado (16).

Em resumo, a tradução e a adaptação do QAD resultaram em um questionário com características e propriedades psicométricas semelhantes às do SDSCA. $\mathrm{O}$ QAD pode, assim, ser considerado um instrumento válido e confiável para medir a aderência ao autocui- dado nos pacientes diabéticos tipo 2 em nosso meio. Estudos futuros com a utilização do QAD em outras amostras e diferentes populações poderão contribuir para seu aprimoramento.

Agradecimentos: À equipe do Ambulatório de Endocrinologia do Hospital Universitário da Universidade Federal de Santa Catarina pela colaboração indispensável na realização deste estudo.

Declaração: os autores declaram não haver conflitos de interesse científico neste estudo.

\section{REFERÊNCIAS}

1. Standards of medical care in diabetes--2009. Diabetes Care. 2009;32 Suppl 1:S13-61.

2. Bilous R. Microvascular disease: what does the UKPDS tell us about diabetic nephropathy? Diabet Med. 2008;25 Suppl 2:25-9.

3. Kohner EM. Microvascular disease: what does the UKPDS tell us about diabetic retinopathy? Diabet Med. 2008;25 Suppl 2:20-4.

4. Toobert DJ, Hampson SE, Glasgow RE. The summary of diabetes self-care activities measure: results from 7 studies and a revised scale. Diabetes Care. 2000;23(7):943-50.

5. Bastos F, Severo M, Lopes C. [Psychometric analysis of diabetes self-care scale (translated and adapted to Portuguese)]. Acta Med Port. 2007;20(1):11-20.

6. Toobert DJ, Glasgow RE. Assessing diabetes self-management: the summary of diabetes self-care activities questionnaire. In: Bradley C, editor. Handbook of Psychology and Diabetes. Switzerland: Harwood Academic; 1994. p. 351-75.

7. Vincent $D, M c E$ wen MM, Pasvogel A. The validity and reliability of a Spanish version of the summary of diabetes self-care activities questionnaire. Nurs Res. 2008;57(2):101-6.

8. Gonzalez JS, Delahanty LM, Safren SA, Meigs JB, Grant RW. Differentiating symptoms of depression from diabetes-specific distress: relationships with self-care in type 2 diabetes. Diabetologia. 2008;51(10):1822-5.

9. Katon WJ, Russo JE, Heckbert SR, Lin EH, Ciechanowski P, Ludman $E$, et al. The relationship between changes in depression symptoms and changes in health risk behaviors in patients with diabetes. Int J Geriatr Psychiatry. 2010;25:466-75.

10. Lin EH, Katon W, Von Korff M, Rutter C, Simon GE, Oliver M, et al. Relationship of depression and diabetes self-care, medication adherence, and preventive care. Diabetes Care. 2004;27(9):2154-60.

11. Reichenheim ME, Moraes CL. [Operationalizing the cross-cultural adaptation of epidemiological measurement instruments]. Rev Saude Publica. 2007;41(4):665-73.

12. Beaton $D E$, Bombardier $C$, Guillemin F, Ferraz MB. Guidelines for the process of cross-cultural adaptation of self-report measures. Spine. 2000;25(24):3186-91.

13. Guillemin F, Bombardier C, Beaton D. Cross-cultural adaptation of health-related quality of life measures: literature review and proposed guidelines. J Clin Epidemiol. 1993;46(12):1417-32.

14. Whittemore R, D'Eramo Melkus G, Grey M. Metabolic control, self-management and psychosocial adjustment in women with type 2 diabetes. J Clin Nurs. 2005;14(2):195-203.

15. Norusis M. SPSS/PC 10.0 base manual. 10.ed. Chicago: SPSS Inc.; 2000.

16. Sacco WP, Malone JI, Morrison AD, Friedman A, Wells K. Effect of a brief, regular telephone intervention by paraprofessionals for type 2 diabetes. J Behav Med. 2009;32(4):349-59. 